\title{
Determinantes del emprendimiento juvenil en Colombia: un análisis desde la nueva economía institucional
}

\author{
TORRES M., MARCELA \\ Vlerick Business School (Colombia) \\ Correo electrónico: marcela.torres@edu.vlerick.com \\ RAMOS R., JosÉ L. \\ Universidad del Norte (Colombia) \\ Correo electrónico: jramos@uninorte.edu.co \\ GALVIS M., MoISÉS M. \\ Centro Emprendimiento Corporación Universitaria Americana (Colombia) \\ Correo electrónico: megalvis@uninorte.edu.co \\ RAMOS C, JosÉ L. \\ Universidad Simón Bolívar (Colombia) \\ Correo electrónico: jose.ramos@unisimonbolivar.edu.co \\ BISWELL J., JUAN E. \\ Universidad del Norte (Colombia) \\ Correo electrónico: jbiswell@uninorte.edu.co
}

\begin{abstract}
RESUMEN
El presente artículo tiene como propósito determinar los factores que intervienen en el emprendimiento de los jóvenes colombianos y estimar el impacto que tienen dichos determinantes sobre la probabilidad de que un joven decida ser un emprendedor. Para lo anterior, se estima un modelo logístico probabilístico alimentado con la base de datos de Global Entrepreneurship Monitor Colombia 2019. Los resultados evidencian que factores informales como la autoconfianza, los marcos de referencia y el nivel educación influyen de manera positiva en la probabilidad de emprender, hecho que evidencia que estos condicionantes informales expresados por North (1993a), son claves para influenciar la iniciativa empresarial por parte de los jóvenes en Colombia. Finalmente, se concluye que las variables de miedo al fracaso, constituidas como condicionantes informales, pueden no ser vistas como una barrera que influya en la probabilidad de ingreso de los jóvenes colombianos en actividades emprendedoras.
\end{abstract}

Palabras clave: emprendimiento, instituciones, jóvenes.

Clasificación JEL: M10; M13; M21; M29.

MSC2010: 91G70; 91B82; 91B84. 


\title{
Determinants of youth entrepreneurship in Colombia: an analysis from the new institutional economy
}

\begin{abstract}
The purpose of this article is to determine the factors involved in the entrepreneurship of Colombian young people and to estimate the impact that these determinants have on the probability that a young person decides to be an entrepreneur. For the above, a probabilistic logistic model fed with the Global Entrepreneurship Monitor Colombia - 2019 database is estimated. The results show that informal factors such as self-confidence, reference frames and education level positively influence the probability of undertaking, a fact that shows that these informal conditions expressed by North (1993a) are key to influencing entrepreneurship by young people in Colombia. Finally, we conclude that the variables of fear of failure, constituted as informal conditioning factors, may not be seen as a barrier that influences the probability of entry of young Colombians into entrepreneurial activities.
\end{abstract}

Keywords: entrepreneurship, institutions, youth.

JEL classification: M10; M13; M21; M29.

MSC2010: 91G70; 91B82; 91B84.

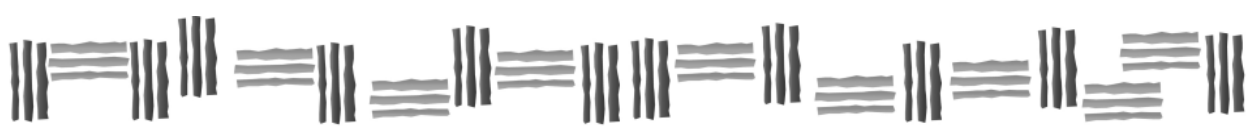




\section{Introducción.}

En los últimos años, el emprendimiento como solución a las bajas tasas de crecimiento económico, se ha convertido en un tema cada vez más estudiado dentro del ámbito académico. En el siglo XVIII, Richard Cantillón definía el concepto de emprendedor y su importancia dentro del desarrollo económico como resultado de la voluntad personal de los individuos. En este mismo sentido, Jean-Baptiste Say, Alfred Marshall y Joseph Schumpeter, elaboraron estudios sobre la configuración del emprendedor como un agente económico relacionado con la creación de empresas (Formichella, 2004).

Estos autores consideraban que el emprendimiento es una iniciativa de tipo económico o social caracterizada por la construcción de oportunidades, innovación y búsqueda de beneficios. Dicha iniciativa es llevada a cabo por la figura del emprendedor, un agente o individuo que busca materializar una idea de negocio con un fin específico a través de sus habilidades personales e ingenio propio, pero sin la seguridad de un retorno seguro sobre la inversión en tiempo o capital que utilice para llevar a cabo su emprendimiento, por causa de la incertidumbre y el riesgo permanente que conlleva perseguir dicho ideal con los medios propios. En este sentido, el emprendedor se configura como un agente económico que transgrede el equilibrio estático planteado por la teoría clásica y que reforma la actividad industrial, impactando el desempeño económico dentro de un territorio (Schumpeter, 1942).

El propósito de este trabajo es identificar los factores asociados al emprendimiento juvenil en Colombia para 2019 y observar cómo se articulan las dinámicas locales con la figura del emprendedor juvenil, ya que la integración de los jóvenes al mercado laboral por medio del emprendimiento es considerada una alternativa útil para superar la pobreza, generar nuevas oportunidades de empleo, disminuir la delincuencia, dinamizar la economía y estimular el desarrollo de experiencias y habilidades (Chigunta, 2002). Así, para efectos de esta investigación, se consideran jóvenes emprendedores aquellos individuos que presentan una iniciativa empresarial y se encuentran en un rango de edad entre los $14 \mathrm{y}$ 26 años, de acuerdo con ley de juventud colombiana (Artículo $3^{\circ}$, ley 375 de 1997).

Ahora bien, según datos del Global Entrepreneurship Monitor (GEM, 2016), el estudio de mayor importancia del emprendimiento realizado en 65 países, reporta que América Latina tiene una tasa de actividad empresarial (TEA: mide la proporción de personas activamente involucradas en la puesta en marcha de iniciativas de autoempleo y emprendimientos) de 18,8\%; mientras que en los países con mayor desarrollo económico la tasa es de $9,1 \%$. De acuerdo con esas cifras, se puede observar, en contraposición con los países desarrollados, un mayor interés en la creación de empresas en los países latinoamericanos. Teniendo en cuenta que los países crecen mediante el desarrollo de un sector estratégico productivo (Industria), las instituciones emergen para apoyar dicha industrialización, incentivando una búsqueda de mayor eficiencia mediante la generación de economías de escala y favoreciendo tanto a las empresas ya establecidas, como al entorno económico para incentivar la creación de empresas por parte de los individuos (GEM, 2008).

Los estudios de GEM han demostrado que la actividad emprendedora presenta variaciones significativas entre los diferentes países que funcionan dentro del marco conceptual de esta medición. Estas variaciones se explican mediante las condiciones del entorno, la capacidad de los individuos para identificar oportunidades, y la aceptación y creación de una cultura emprendedora. Adicionalmente, GEM ha evidenciado que la actividad emprendedora se relaciona positivamente con el crecimiento económico, y esta relación difiere según la fase de desarrollo en la que se encuentra el país (Acs \& Amorós, 2008). De este modo, y en la perspectiva de indagar sobre los factores determinantes del emprendimiento juvenil en Colombia, en este estudio se utilizaron variables perceptuales, conductuales y sociodemográficas contenidas en GEM.

El artículo está dividido en cinco partes. La primera, comprendida por la presente introducción. La segunda parte incluye la evidencia empírica de los estudios relacionados con el emprendimiento juvenil. La tercera sección contiene los fundamentos teóricos de la economía institucional relacionados con las variables de estudio. En el cuarto apartado se presenta la metodología, las variables y el modelo econométrico que permitió determinar los factores asociados al emprendimiento juvenil en Colombia. 
Finalmente, se registran los resultados con sus interpretaciones y contraste con los preceptos teóricos de la economía institucional, lo que permitió generar las conclusiones importantes sobre el emprendimiento juvenil.

\section{Revisión de la literatura.}

Los trabajos realizados en los últimos años evidencian la relevancia que tiene el estudio del emprendimiento en los jóvenes, destacándose factores que influyen tanto positiva, como negativamente en el deseo de éstos por llevar a cabo actividades de tipo empresarial. El emprendimiento es considerado como una alternativa para superar el desempleo juvenil en un territorio, el cual impacta el dinamismo económico y, en consecuencia, el bienestar de una sociedad (Maxwell, 2002; García, 2016; Yáñez \& Escareño, 2020; Nazri et al., 2016). Adicionalmente, esta población suele asumir un rol de agentes de cambio a través del emprendimiento social (Bosma et al, 2016; Makua, Amigo \& Doistua, 2017), convirtiéndose en el foco de atención para el diseño de una política pública de empleo.

En esta primera parte, se expone la evidencia empírica recopilada como fuente para identificar y caracterizar las variables explícitas en el modelo econométrico; en particular, de tipo social, económico, demográfico y psicológico, todas ellas inherentes a cada individuo y al ambiente que los rodea. Igualmente, se identifican aquellas que determinan la probabilidad de emprender en la población joven.

Dentro de los factores sociales, por ejemplo, se destacan las variables de percepción de riesgo, la delincuencia y el miedo al fracaso como elementos que tienden a generar en los emprendedores juveniles un impacto significativamente negativo frente a la probabilidad de que éstos hagan parte de procesos de emprendimiento (Fairlie, 2002; Lévesque \& Minniti, 2006; Espí et al., 2007; Olmos \& Castillo, 2009; Olawale, 2010; Lafuente, Vaillant \& Gómez, 2007).

Por su parte, Blanchflower y Meyer (1994) consideran que el nivel de educación del individuo es un factor social que impacta positivamente el emprendimiento juvenil. Esta consideración también la comparten las investigaciones realizadas por Rasheed (2000), Turker y Selcuk (2008), Pihie (2009), Montes y Siga (2009) y Capaldo (1997). De igual manera, Henderson y Robertson (1999) considera que el desenvolvimiento de los jóvenes en un ambiente académico que incentive el emprendimiento afecta positivamente su disposición ante la creación de una empresa.

En este sentido, Schiller y Crewson (1997), Lafuente, Vaillant, y Gómez (2007), Turker y Selcuk (2008), Pihie (2002), Olmos y Castillo (2009), Sahasranamam y Nandakumar (2020) consideran que la relación de los jóvenes con un entorno social emprendedor, en donde éstos tienen la posibilidad de conocer directa o indirectamente una iniciativa empresarial por parte de un familiar o allegado a la familia, impacta positivamente en la creación de modelos de referencia, hecho que aumenta la probabilidad de que el joven sea emprendedor.

Dentro de los factores de tipo económico y demográfico, las dificultades de acceso al capital, financiación y los bajos ingresos que posean los individuos jóvenes detraen la probabilidad de generación de emprendimientos (Blanchflower \& Oswald, 1990; Henderson y Robertson, 1999; Espí et al., 2007; Turker \& Selcuk, 2008; Olawale, 2010; Capaldo, 1997; Fairlie, 2002). Mientras que contar con un soporte económico, como por ejemplo una herencia, afecta positiva y significativamente la iniciativa empresarial (Blanchflower \& Oswald, 1990).

Las investigaciones de Blanchflower y Meyer (1994), Blanchflower y Oswald (1998) y Schiller y Crewson (1997) apuntan a que los factores demográficos (edad del individuo) tienen una relación positiva en los emprendedores juveniles. Es posible encontrar soporte a este resultado si observamos dentro de la literatura la variable de experiencia laboral, que en estudios realizados por Henderson y Robertson (1999), Lévesque y Minniti (2006) posee un impacto positivo y significativo dentro de la iniciativa empresarial juvenil. 
Por otro lado, el género también se destaca como un factor que afecta dicha iniciativa. Wilson, Marlino y Kickul (2004), y Wilson, Kickul, y Marlino (2007), encuentran que ser hombre aumenta la probabilidad de realizar emprendimientos. Por su parte, Schiller y Crewson (1997) examinan el impacto del estado civil de los jóvenes, sugiriendo que los hombres solteros tienen mayor probabilidad de ser emprendedores, aunque para el contexto mexicano Canales, Román y Ovando (2017) encuentran que los jóvenes casados adquieren mayor presencia en la actividad emprendedora, sin enfatizar si esta participación es por oportunidad o necesidad.

Adicionalmente, Henderson y Robertson (1999), Rasheed (2000), Thomas (2009), Olmos y Castillo (2009), examinan la influencia de factores psicológicos usando variables tales como el control del locus interno y la tolerancia a las adversidades, encontrando como resultado que los jóvenes que poseen altos niveles de tolerancia y de control poseen una tendencia mayor a las iniciativas empresariales, contrariamente a los jóvenes que no poseen dichas características.

Por otra parte, Wilson, Marlino y Kickul (2007), Lafuente, Vaillant y Gómez (2007), y Capaldo (1997), consideran en sus análisis variables como la autoconfianza, la percepción de habilidades por parte del individuo y la autoeficacia, generando indicios de que éstas afectan positivamente la creación de empresas en la población joven. Olawale (2010), Wilson, Marlino y Kickul (2004) y Fairlie (2002), analizan la autonomía y la búsqueda de independencia como variables que influyen los emprendimientos. Por otro lado, Espí et al. (2007) indican que la posibilidad de poner en práctica ideas propias, la independencia personal y trabajar con mayor libertad de decisión y de acción, constituyen factores que inducen el autoempleo.

De acuerdo con la literatura teórica y empírica citada anteriormente, se evidencia que entre los factores que incentivan el emprendimiento juvenil destacan: la educación del individuo, la edad, el género, los modelos de referencia, la autonomía, la autoeficacia, el control de locus interno y las habilidades empresariales.

Dentro de los factores que se constituyen en un óbice para el empresario joven, se encuentran factores como las leyes del gobierno, la falta de apoyo estructural, la escasez de capital inicial y la financiación bancaria. Al igual que las variables inherentes a los individuos, como la hostilidad, las dificultades de comunicación, la baja autoconfianza, el riesgo elevado y el miedo al fracaso y la delincuencia.

\section{Argumentos teóricos.}

Los preceptos utilizados para contrastar y analizar los resultados del modelo econométrico, están centrados en la teoría institucionalista.

\subsection{Teoría institucional.}

La teoría económica institucional nace a finales del siglo XIX y se configura como una vertiente alternativa a la economía ortodoxa, que apunta a la comprensión de la historia, la cultura y los pensamientos que conforman las conductas humanas, afectando el origen y evolución de las instituciones. Es así como surge una comprensión descriptiva generalizada del orden económico y sus fenómenos, teniendo en cuenta como principal influencia las instituciones, entendidas como métodos o modelos de comportamientos seguidos por la sociedad (Hamilton, 1919).

Remontándonos al proceso científico que ha tenido la economía institucional, se evidencia una segmentación dentro dicha escuela pensamiento económico, que siguiendo a Parada (2003), se divide en dos vertientes principalmente: Economía Original Institucional (EIO), representada por Thorstein Veblen, Wesley Mitchell, John Commons, John Clark, Clarence Ayres y la Nueva Economía 
Institucional (NIE) impulsada por Ronald Coase (1937), Douglas North y Oliver Williamson, entre otros.

A pesar de la falta de unanimidad conceptual en la escuela institucional, se distinguen dos principios básicos que permiten su conjugación (Urbano, Díaz \& Hernández, 2007). El primero presupone una relación dinámica y constante con las instituciones socio-culturales y políticas haciendo difícil la separación de los procesos económicos de los procesos sociales; y el segundo, reconoce una ruptura con los conceptos de equilibrio estático planteado por la escuela neoclásica, destacando la creencia de procesos evolutivos condicionados por el comportamiento de las personas Urbano, Díaz \& Hernández, 2007).

Dada esta distinción, el presente análisis económico se basa en los planteamientos de la Nueva Economía Institucional, particularmente en las ideas de Douglas North.

\subsection{Enfoque institucional de Douglas North.}

Siguiendo la propuesta de Urbano, Díaz y Hernández (2007), los planteamientos de North son útiles para estudiar los factores que determinan la creación de empresas, ya que: (a) Dicha teoría genera un entendimiento de las variables que delimitan e incentivan el proceso económico mediante el comportamiento humano; y (b) La creciente evidencia empírica que soporta el emprendimiento por medio de un marco teórico institucional (Nee \& Young, 1991; Veciana, 1988; Aponte, 2002; Freytag \& Thurik, 2006; Martínez et. al., 2010).

Al respecto, North (1993a) realiza una evaluación sobre la naturaleza de las instituciones y el cambio institucional, considerando la historia y la cultura como los principales determinantes del desempeño social y económico evidenciado en los diferentes países, suponiendo agentes con racionalidad limitada e información imperfecta. De esta forma, la configuración de la conducta humana y los costes de transacción, junto a algunos otros supuestos, construyen un marco analítico en el que las instituciones son definidas por el autor como imposiciones ideadas por los seres humanos que estructuran y limitan sus interacciones, reduciendo la incertidumbre mediante la construcción de una estructura de interacción de hábitos, reglas, costumbres, y políticas que determinan las posibles elecciones por parte de los individuos (North, 1993a; 1993b; 1994).

Dentro de dicho marco analítico, North (1993b) define a las organizaciones u organismos como "grupos de individuos unidos por un propósito común con el fin de lograr ciertos objetivos" (p. 3), e incluye cuatro tipos de organizaciones, a saber, las políticas, las económicas, las sociales y las educativas. Además de esto, North (1993a) considera que las organizaciones se ven condicionadas por el marco institucional que las regula y la interacción humana que las rodea. Siendo de esta manera como nacen las limitaciones de tipo formal e informal que configuran las reglas de juego.

Definiendo estas limitaciones, se tiene que las de tipo formal están constituidas por normas, leyes, constituciones o reglamentos; y las de tipo informal se constituyen por códigos de conducta y comportamientos, hábitos, creencias, valores sociales y actitudes. Para este último tipo, se especifican las siguientes limitaciones: (a) las que son extensiones, interpretaciones y modificaciones de normas formales; (b) las normas de conducta sancionadas socialmente; y (c) las normas de conducta aceptadas internamente (Urbano, Díaz \& Hernández, 2007).

Considerando lo anterior, podemos concluir que existen dos vías principales mediante las que se ve afectada la constitución del sistema de instituciones dentro de una sociedad. La primera son las limitaciones formales, que contienen pautas políticas, medidas judiciales, reglas de tipo económico y son fácilmente identificables por medio de la generación de decretos, leyes, y/o manuales escritos. La segunda son las limitaciones informales, que se constituyen a partir de valores y comportamientos sociales e históricos, muy poco reconocidos mediante pautas o manuales escritos. 
Al respecto, North (1993a) argumenta que la existencia de limitaciones de tipo formal es desarrollada para fomentar y complementar la eficacia del desempeño económico de una región al disminuir los costes de información o al sustituir reglas informales que afecten negativamente dicho desempeño. Estos ajustes marginales realizados al marco institucional por medio de variaciones a los tipos de limitaciones son denominados por el autor como un cambio institucional.

Este cambio, es un proceso constante, producto de variaciones, tanto de las limitaciones formales (modificación de las reglas económicas o políticas) como de las informales (decisiones que toman los agentes). En este sentido, North (1993a) afirma que este proceso constante permite la interacción entre instituciones y agentes participantes de la economía u organizaciones, generándose cambios continuos y evolutivos en el desempeño económico y social que se presenta dentro de un límite geográfico (Urbano, Díaz \& Hernández, 2007).

Así pues, el marco analítico del cambio institucional propuesto por North (1993a), citado por Urbano, Díaz \& Hernández (2007) consta de cinco proposiciones decisivas:

(1) La interacción entre instituciones y organizaciones, es la clave para el cambio institucional. (2) La competencia direcciona a las organizaciones a invertir en conocimiento para competir. (3) El marco institucional provee los incentivos para tener máximas retribuciones. (4) Las percepciones vienen determinadas por las estructuras mentales de los emprendedores, y (5) Las economías de escala, son las que determinan el cambio institucional... (p. 22)

\section{Instituciones y espíritu empresarial.}

Con base en la teoría institucional de North (1993a), se realiza una vinculación entre dicha teoría y algunos estudios en torno a la creación de empresas que enfatizan en el espíritu empresarial de los jóvenes, teniendo como referencia la modelación realizada por Bruton, Ahlstrom y Li (2010). La teoría institucional dirige su análisis al estudio del impacto dentro de la economía, considerando la existencia de reglas, hábitos, costumbres, normas que rigen la conducta de los individuos e impactan de igual manera la creación de instituciones. Este entendimiento nos ayuda a comprender cómo el entorno en el que se desenvuelven los jóvenes impacta e influye, tanto en las decisiones tomadas por éstos como en las organizaciones de tipo económico, social y político existentes dentro de la sociedad de la que ellos forman parte.

Es así, como Bruton, Ahlstrom y Li (2010) sugieren que: "el entorno institucional ejerce una poderosa influencia no sólo sobre las tasas de entrada a las organizaciones de tipo económicas, sino también en las trayectorias resultantes de las iniciativas empresariales" (p. 23). Los autores plantean una relación entre las fuerzas institucionales y el espíritu empresarial, por medio de tres categorías institucionales.

La primera, denominada la base reguladora, representa un modelo racional de comportamiento basado en sanciones y valores tradicionales de una sociedad. De esta manera, se configura a las instituciones como guías de la conducta humana, mediante la conformación de reglas de juego, su monitoreo y aplicación. Dichas reglas son esenciales a la hora de brindar a los emprendedores nacientes directrices para el cumplimento de las leyes o reacciones ante la carencia de leyes dentro de cierta región.

La segunda categoría está comprendida por la base normativa, representada por modelos organizacionales y de conducta integradas por dimensiones obligatorias de tipo social, de interacción organizacional y profesional. Dentro de estas dimensiones, las instituciones actúan como limitantes de normas de comportamiento aceptadas y/o esperadas en situaciones sociales, comerciales y legales. Se encuentran normas que facilitan y promueven el emprendimiento y su respectiva financiación; como 
también normas que desincentivan la iniciativa empresarial mediante barreras legales, o regulaciones elevadas.

Finalmente, la base cognitiva representa un modelo de comportamiento individual y subjetivo, basado en la construcción de reglas derivadas de la cultura e interacción humana que limitan las creencias, hábitos y acciones apropiadas seguidas por una sociedad. Este pilar es considerado de mucha relevancia dentro del espíritu empresarial, debido a que es importante entender las actitudes de la sociedad ante la iniciativa empresarial y el posible aliento que dichas creencias pueden generar sobre el deseo de emprender por parte de los jóvenes.

Utilizando la anterior categorización, se incluye la base reguladora y normativa dentro de las limitaciones formales, mientras que la base cognitiva de las instituciones forma parte de las limitaciones de tipo informal que pueden llegar a moldear las conductas de los individuos y por ende el comportamiento de los jóvenes. Esta influencia es percibida por medio de factores que nutren el entorno de los jóvenes emprendedores. De acuerdo con North (1993b), dichas variables se clasifican en:

(1) Condicionantes formales: comprendidas por la creación de incentivos por parte del gobierno, la facilidad de acceso a recurso de financiación, disponibilidad de capital, legitimidad y programa de educación en emprendimiento, y

(2) Condicionantes informales: comprendidas por las creencias y actitudes que poseen los individuos ante los empresarios, la estabilidad macroeconómica, las motivaciones ante la iniciativa empresarial, expectativas y aspiraciones inherentes a cada individuo dentro de una sociedad, que ayudan a conjugar el espíritu empresarial dentro de éstos (Foster, 1986; Baumol et al., 2009; Soto, 2000).

Figura 1. Fuerzas institucionales sobre emprendimiento juvenil.

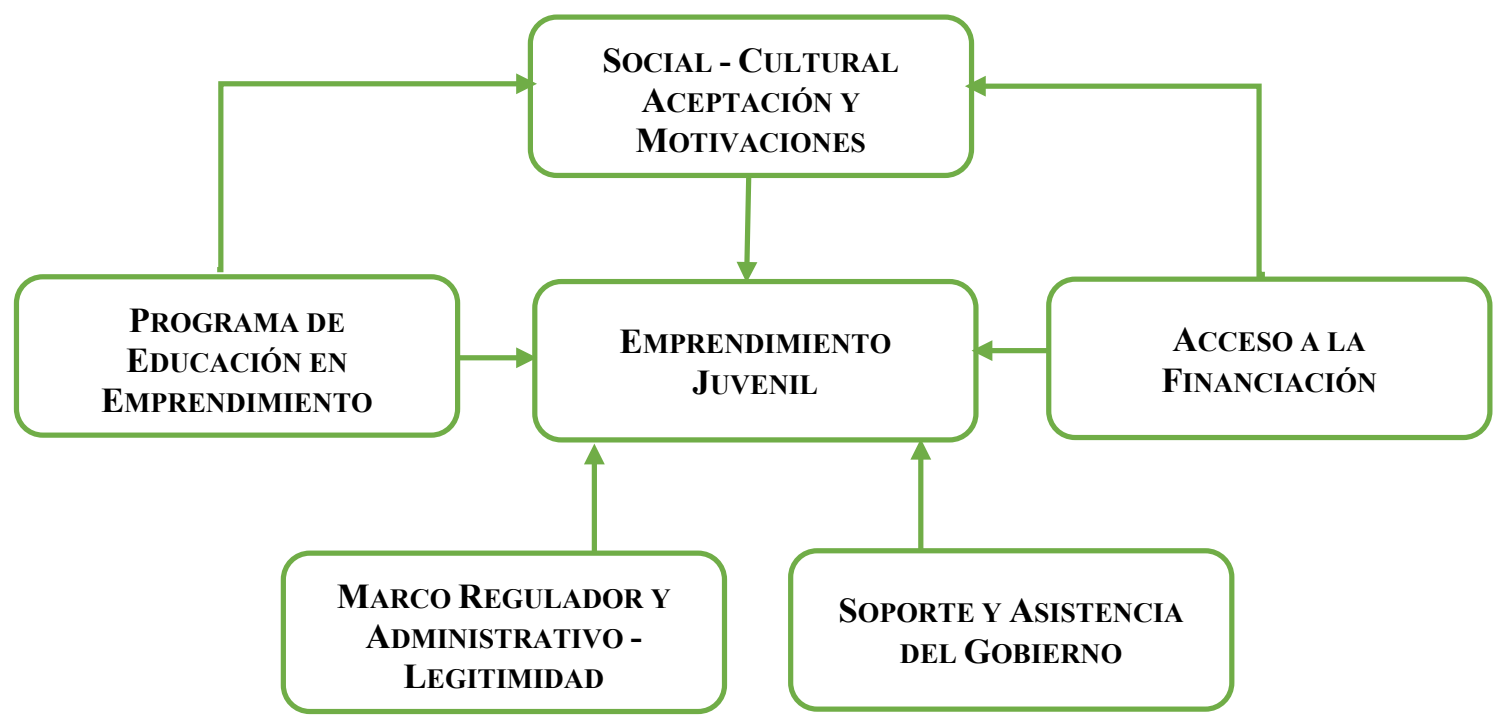

Fuente: Adaptado de Schoof (2006).

El Informe Nacional sobre Emprendimiento Juvenil en Colombia destaca que los factores institucionales determinantes del emprendimiento juvenil están relacionados con las condiciones sociales de los jóvenes emprendedores. Así, de acuerdo con Montes y Jenny (2016):

El capital humano, las políticas y regulaciones, las condiciones de demanda y el ambiente cultural propicio para los negocios de valor agregado, son las principales fortalezas de Colombia para emprender negocios, mientras 
que las debilidades están en las condiciones sociales, el capital social, las plataformas de ciencia y tecnología, el acceso a financiamiento y la estructura empresarial. (p. 9)

Considerando los anteriores elementos teóricos, se presentan las siguientes hipótesis de investigación, que son analizadas en la sección de análisis de los resultados:

Tabla 1. Hipótesis de estudio: condicionantes formales e informales.

\begin{tabular}{|c|c|c|}
\hline HIPÓTESIS & CONDICIONANTES FORMALES & $\begin{array}{l}\text { AUTORES } \\
\end{array}$ \\
\hline H1 & $\begin{array}{l}\text { La probabilidad de ser un joven } \\
\text { emprendedor disminuye frente a la } \\
\text { dificultad para acceder a capital líquido. }\end{array}$ & $\begin{array}{l}\text { Blanchflower \& Oswald (1998), Henderson } \\
\text { (1999), Espí et al. (2007), Turker \& Selcuk } \\
\text { (2008), Olawale (2010), Capaldo (1997). }\end{array}$ \\
\hline H2 & $\begin{array}{l}\text { La probabilidad de ser un joven } \\
\text { emprendedor se incrementa con el nivel } \\
\text { de educación. }\end{array}$ & $\begin{array}{l}\text { Blanchflower \& Meyer (1994), Rasheed } \\
\text { (2000), Turker \& Selcuk (2008), Pihie (2009), } \\
\text { Montes \& Siga (2009), Capaldo (1997), Shah, } \\
\text { Amjed \& Jacob (2020). }\end{array}$ \\
\hline HIPÓTESIS & CONDICIONANTES INFORMALES & AUTORES \\
\hline H3 & $\begin{array}{l}\text { La probabilidad del emprendedor } \\
\text { aumenta para quienes perciben } \\
\text { oportunidades en su entorno. }\end{array}$ & $\begin{array}{l}\text { Blanchflower \& Meyer (1994), Kirzner (1973), } \\
\text { Arenius \& Minniti (2005). }\end{array}$ \\
\hline H4 & $\begin{array}{l}\text { La probabilidad de ser un joven } \\
\text { emprendedor disminuye ante un } \\
\text { aumento en la percepción de riesgo. }\end{array}$ & $\begin{array}{l}\text { Fairlie (2002), Lévesque \& Minniti (2006), } \\
\text { Espí et al. (2007), Olmos \& Castillo (2009), } \\
\text { Olawale (2010), Lafuente, Vaillant \& Gómez } \\
\text { (2007), García (2016). }\end{array}$ \\
\hline H5 & $\begin{array}{l}\text { La autoconfianza incrementa la } \\
\text { probabilidad de ser un joven } \\
\text { emprendedor. }\end{array}$ & $\begin{array}{l}\text { Fairlie (2002), Delgado et al. (2007), Wilson, } \\
\text { Kickul \& Marlino (2007), Lafuente, Vaillant \& } \\
\text { Gómez (2007), Olawale (2010), Capaldo } \\
\text { (1997), Schiller \& Crewson (1997), Henderson } \\
\text { (1999), Wilson, Marlino \& Kickul (2004) } \\
\text { Shah, Amjed \& Jaboob (2020). }\end{array}$ \\
\hline H6 & $\begin{array}{l}\text { Los jóvenes tienen mayor probabilidad } \\
\text { de emprender un negocio que las } \\
\text { jóvenes. }\end{array}$ & $\begin{array}{l}\text { Blanchflower \& Oswald (1990), Blanchflower } \\
\text { \& Meyer (1994), Blanchflower \& Oswald } \\
\text { (1998), Wilson, Marlino \& Kickul (2004), } \\
\text { Wilson, Kickul, \& Marlino (2007), Schiller \& } \\
\text { Crewson, (1997). }\end{array}$ \\
\hline H7 & $\begin{array}{l}\text { Ante la existencia de mayores modelos } \\
\text { de referencias, la probabilidad de ser } \\
\text { un joven emprendedor es mayor. }\end{array}$ & $\begin{array}{l}\text { Blanchflower \& Oswald (1990), Schiller \& } \\
\text { Crewson (1997), Lafuente, Vaillant, \& Gómez } \\
\text { (2007), Turker \& Selcuk (2008), Pihie (2009), } \\
\text { Olmos \& Castillo (2009). }\end{array}$ \\
\hline H8 & $\begin{array}{l}\text { A mayor valoración del } \\
\text { emprendimiento en la sociedad, mayor } \\
\text { será la probabilidad de emprender en los } \\
\text { jóvenes. }\end{array}$ & $\begin{array}{l}\text { Shapero \& Sokol (1982), Veciana (1988), } \\
\text { Shah, Amjed \& Jaboob (2020). }\end{array}$ \\
\hline
\end{tabular}

Fuente: Elaboración propia.

Este conjunto de hipótesis reúne la perspectiva de diversos autores y considera un matiz de posibilidades tanto positivas como negativas que se asocian a las probabilidades de un mayor emprendimiento juvenil. De las anteriores hipótesis, se deriva el siguiente modelo teórico (Figura 2), que orientó las respuestas a las mismas. 
Figura 2. Modelo teórico condicionantes formales e informales de la iniciativa emprendedora juvenil.

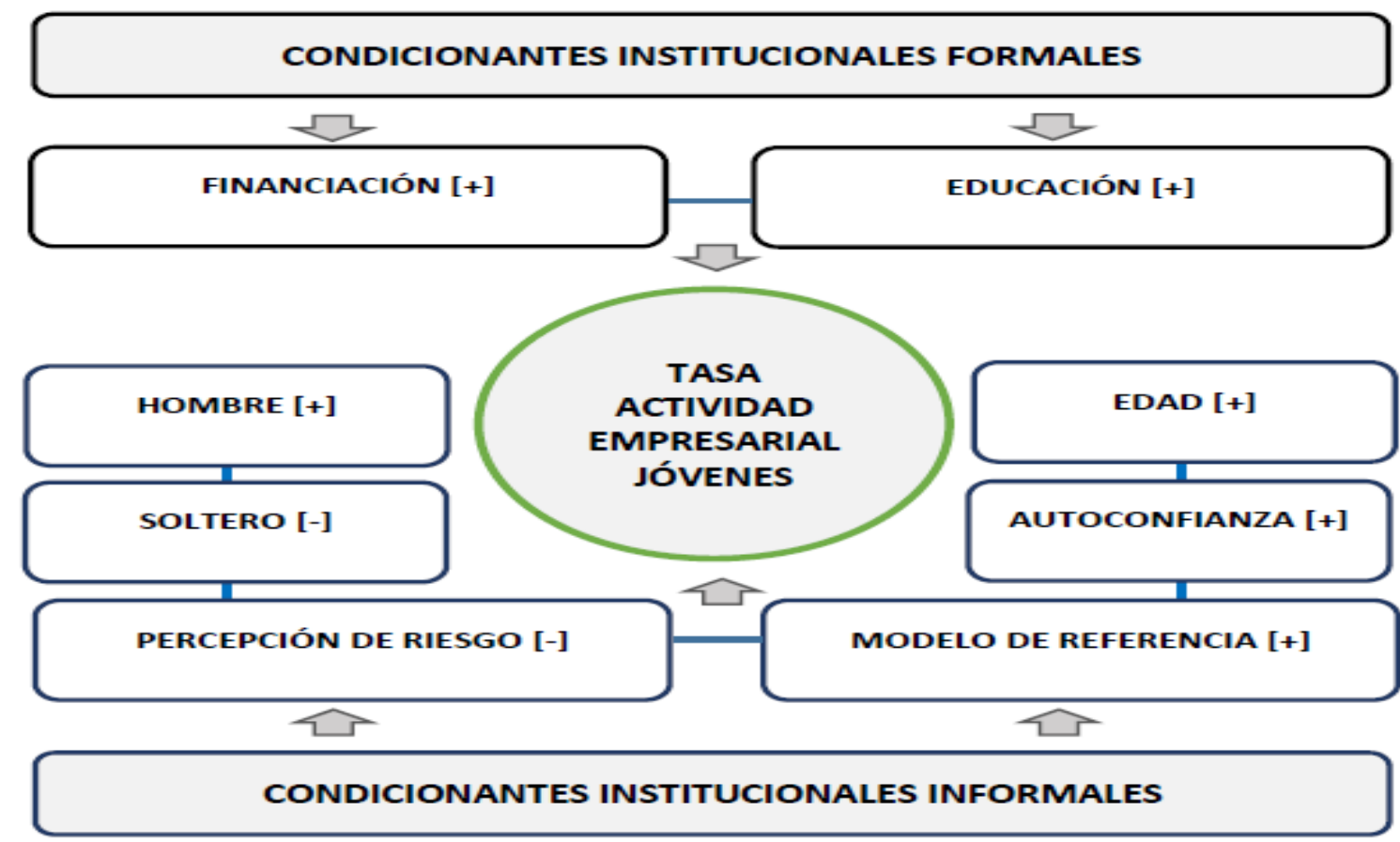

Fuente: Elaboración propia.

\section{Variables y modelo econométrico.}

Para dar respuesta a las ocho hipótesis, se utilizó la base de datos de GEM Colombia - 2019. La muestra se seleccionó teniendo en cuenta la definición de la juventud plasmada en la ley 375 de 1994 del Ministerio de Educación Nacional de Colombia, por lo que se evalúan 465 individuos pertenecientes a un rango de edad entre 18 y 26 años. La operacionalización de las variables recogidas se reporta en la siguiente tabla.

Tabla 2. Operacionalización de variables.

\begin{tabular}{|c|c|c|}
\hline VARIABLES INDEPENDIENTES & VARIABLES GEM & CATEGORÍAS \\
\hline \multirow[t]{2}{*}{$\begin{array}{c}\text { Condicionantes Institucionales } \\
\text { Formales }\end{array}$} & Ingreso del Hogar (Cohhinc $\left.\left[{ }^{*}\right]\right)$ & $\begin{array}{l}1=\text { Entre } 1 \text { a } 2 \text { SMMLV } \\
2=\text { Entre } 2 \text { a } 3 \text { SMMLV } \\
3=\text { Entre } 3 \text { a } 4 \text { SMMLV } \\
4=\text { Entre } 4 \text { a } 5 \text { SMMLV } \\
5=\text { Entre } 5 \text { a } 6 \text { SMMLV } \\
6=\text { Más } 6 \text { SMMLV }\end{array}$ \\
\hline & Nivel Educativo (Coreduc) & $\begin{array}{l}1=\text { Sin estudios } \\
2=\text { Técnico } \\
3=\text { Tecnólogo } \\
4=\text { Universitario }\end{array}$ \\
\hline \multirow{2}{*}{$\begin{array}{c}\text { Condicionantes Institucionales } \\
\text { Informales }\end{array}$} & Miedo al Fracaso (Fearfail $[* *])$ & $\begin{array}{l}0=\text { No } \\
1=\text { Sí }\end{array}$ \\
\hline & $\begin{array}{l}\text { Percepción de Habilidades y } \\
\text { Conocimientos en Emprendimiento } \\
\left(\text { Suskill }\left[{ }^{* *}\right]\right)\end{array}$ & $\begin{array}{l}0=\mathrm{No} \\
1=\mathrm{Sí}\end{array}$ \\
\hline
\end{tabular}




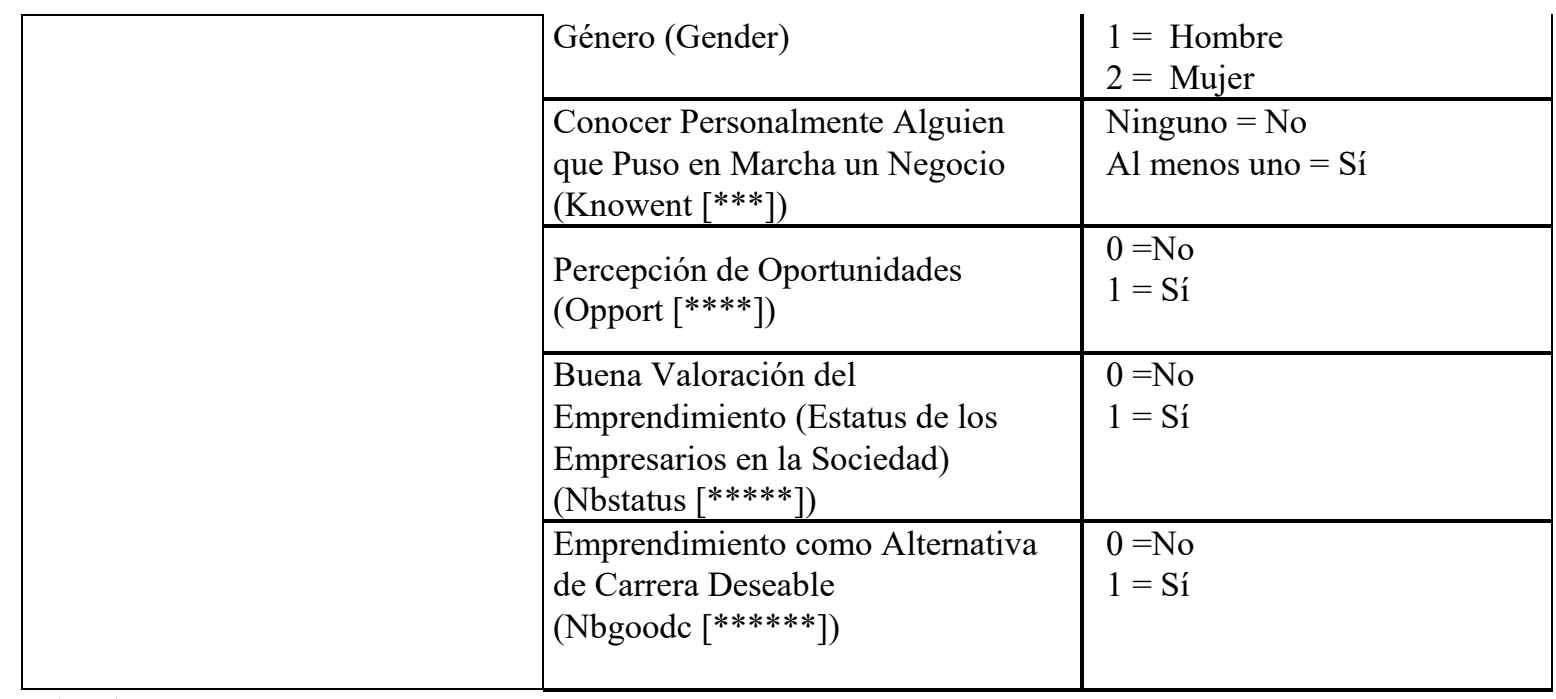

\section{VARIABLE DEPENDIENTE}

\begin{tabular}{|c|l|}
\hline Tasa Actividad Temprana Empresarial Jóvenes (Teajovenesrc) & $\begin{array}{l}0=\text { No } \\
1=\text { Sí }\end{array}$ \\
\hline
\end{tabular}

Nota: SMMVL hace referencia a Salario Mínimo Mensual Legal Vigente.

[*] La variable capital líquido se midió con la proxy de ingresos del hogar, que es una variable categórica medida en rangos de ingreso.

[**] La percepción del riesgo se midió a través de la variable proxy miedo al fracaso.

[***] La variable autoconfianza se midió a través de la percepción de habilidades y conocimientos en emprendimiento.

[****] El modelo de referencia se midió a través de la variable proxy 'conocer a alguien cercano que sea emprendedor'. La variable de percepción de oportunidades se midió preguntando directamente sobre la percepción de oportunidades del individuo en la zona.

[*****] La variable de estatus de los empresarios se midió mediante la variable proxy de "la sociedad percibe a los emprendedores exitosos con un alto estatus".

[******] La variable de emprendimiento como alternativa de carrera deseable se midió mediante la pregunta directa de esta percepción a los individuos.

[*******] La variable TEA se refiere la proporción de jóvenes que están vinculados activamente en el desarrollo y/o puesta en marcha de un emprendimiento.

Fuente: Elaboración propia.

Por su parte, la Tabla 3 recoge las variables descriptivas que permiten identificar el perfil socio económico de los jóvenes colombianos. De esta forma, se revela que las características socio económicas de los individuos evaluados están constituidas de la siguiente manera: la mayoría de los jóvenes incluidos dentro de la muestra pertenecen al género femenino (51.61\%); cuentan con un nivel de educación bajo, sólo primaria y secundaria (64.08 \%); y hacen parte de un hogar cuyos ingresos oscilan entre COP $\$ 737.717$ y COP $\$ 1.475 .434$ en promedio $(62.79 \%)(\mathrm{COP}=$ Pesos Colombianos. Aproximadamente corresponde a 163 y 326 euros, respectivamente. Tasa de octubre 10 de $2020=4.528$ euros por $\mathrm{COP}$ ).

Los jóvenes colombianos cuentan con un perfil caracterizado por poca incertidumbre, donde el $62.58 \%$ de los mismos, dicen no poseer miedo al fracaso; así se infiere que la percepción del riesgo por parte de los jóvenes no es elevada. Ante la variable de auto confianza, los datos registran que el $59.35 \%$ de los individuos perciben tener habilidades y conocimientos en emprendimiento. Por otro lado, el $37.58 \%$ de los jóvenes no cuentan con un modelo de referencia. Adicionalmente, menos de la mitad de los jóvenes colombianos perciben que habrá oportunidades para emprender en los próximos seis meses (43.01\%). Por último, el $61.72 \%$ de los individuos evaluados consideran el emprendimiento como una 
alternativa de carrera deseable, mientras que el $61.51 \%$ percibe que los emprendedores exitosos gozan de un alto estatus en la sociedad.

Tabla 3. Perfil Socioeconómico del emprendimiento juvenil.

\begin{tabular}{|c|c|c|c|}
\hline \multicolumn{2}{|l|}{ VARIABLES } & NÚMERO & PORCENTAJE \\
\hline \multirow{7}{*}{ Ingreso del Hogar (Cohhinc) } & $\begin{array}{l}\text { Menos de } 1 \\
\text { SMMLV }\end{array}$ & 58 & $12.47 \%$ \\
\hline & Entre 1 y 2 SMMLV & 234 & $50.32 \%$ \\
\hline & Entre 2 y 3 SMMLV & 92 & $19.78 \%$ \\
\hline & Entre 3 y 4 SMMLV & 53 & $11.40 \%$ \\
\hline & Entre 4 y 5 SMMLV & 22 & $4.73 \%$ \\
\hline & Entre 5 a 6 SMMLV & 6 & $1.29 \%$ \\
\hline & Más de 6 SMMLV & 0 & $0 \%$ \\
\hline \multirow{5}{*}{ Nivel Educativo (Coreduc) } & $\begin{array}{l}\text { Primaria y } \\
\text { secundaria }\end{array}$ & 298 & $64.09 \%$ \\
\hline & Técnico & 90 & $19.35 \%$ \\
\hline & Tecnólogo & 40 & $8.60 \%$ \\
\hline & Universitario & 32 & $6.88 \%$ \\
\hline & Postgrado & 5 & $1.80 \%$ \\
\hline \multirow{2}{*}{$\begin{array}{l}\text { Habilidades y Conocimientos en } \\
\text { Emprendimiento (Suskil19) }\end{array}$} & Sí & 276 & $59.35 \%$ \\
\hline & No & 159 & $40.65 \%$ \\
\hline \multirow{2}{*}{ Modelo de Referencia (Knowen19) } & Ninguno & 174 & $37.58 \%$ \\
\hline & Al menos uno & 294 & $49.41 \%$ \\
\hline \multirow{2}{*}{ Miedo al fracaso (Fearfail19) } & Sí & 174 & $37.42 \%$ \\
\hline & No & 291 & $62.58 \%$ \\
\hline \multirow{2}{*}{ Género (Gender) } & Hombre & 225 & $48.39 \%$ \\
\hline & Mujer & 240 & $51.61 \%$ \\
\hline \multirow{2}{*}{ Percepción de Oportunidades(Opport19) } & Sí & 200 & $43.01 \%$ \\
\hline & No & 265 & $56.99 \%$ \\
\hline \multirow{2}{*}{ Alto Estatus de los Empresarios(Nbstat19) } & Sí & 286 & $61.51 \%$ \\
\hline & No & 179 & $38.49 \%$ \\
\hline \multirow{2}{*}{ Opción de Carrera Deseable (Nbgood19) } & Sí & 287 & $61.72 \%$ \\
\hline & No & 168 & $38.28 \%$ \\
\hline
\end{tabular}

Fuente: Elaboración propia de los autores, 2020 con base en GEM 2019.

\section{Modelo econométrico.}

Dada la naturaleza binaria de la variable dependiente que se analiza, se considera conveniente la estimación de un modelo de elección binaria, sea Logit o Probit. En forma general, modelos de este tipo se pueden expresar en términos de una variable latente, $y_{i}^{*}$, como:

$$
\begin{gathered}
y_{i}^{*}=x_{i} \beta_{i}+\mu_{i} \\
y_{i}=\left\{\begin{array}{l}
0 \text { si } y_{i}^{*}<0 \\
1 \text { si } y_{i}^{*} \geq 0
\end{array}\right\}
\end{gathered}
$$

donde $y_{i}^{*}$ se relaciona linealmente al conjunto de factores $\mathbf{X}$ y a un proceso de error estocástico $\mu$. Haciendo uso de (1) y (2) se logra modelar la probabilidad de un individuo de llevar a cabo cada elección.

$$
P_{r}\left(y^{*}>0 \mid \mathrm{X}\right)=
$$




$$
\begin{gathered}
P_{r}(\mu>-x \beta \mid \mathrm{X})= \\
P_{r}(\mu<x \beta \mid \mathrm{X})= \\
P_{r}(y=1 \mid \mathrm{X})=\Psi\left(y_{i}^{*}\right)
\end{gathered}
$$

donde $\Psi($.$) es una función de distribución acumulada. Cuando \Psi($.$) corresponde a la función$ de distribución normal, se estima un modelo Probit. Entretanto, el modelo Logit hace uso de la función de distribución acumulada de la distribución logística:

$$
P_{r}(y=1 \mid \mathrm{X})=\frac{\exp (x \beta)}{1+\exp (x \beta)}
$$

Tradicionalmente, se emplea el criterio de información Akaike y Schwarz, para definir qué modelo resulta más apropiado. Este criterio penaliza la cantidad de parámetros de cada uno de los modelos basándose en el principio de parsimonia del modelo, es decir, es un criterio que toma la bondad de ajuste del modelo a los datos a través de la función objetivo de máxima verosimilitud al tiempo que tiene en cuenta una penalización a la cantidad total de parámetros del modelo (Akaike, 1973). De esta manera, siguiendo los elementos teóricos comentados, la especificación del modelo a estimar corresponde a:

$$
\begin{array}{r}
\text { Teajovenesrc }=\beta_{0}+\beta_{1} \text { Cohhinc }+\beta_{2} \text { Coreduc }+\beta_{3} \text { Fearfail }+\beta_{4} \text { Suskill }+\beta_{5} \text { Gender }+\beta_{6} \text { Knowent }+ \\
\beta_{7} \text { Opport }+\beta_{8} \text { Nbstatus }+\beta_{9} \text { Nbgood }+\mu
\end{array}
$$

La variable dependiente del modelo binario es la participación en la Tasa de Actividad Emprendedora Juvenil (Teajovenesrc), que está definida por GEM como la tasa de prevalencia de las personas en la población en edad de trabajar que participan activamente en la creación de empresa; dentro de la medida se incluye tanto a los empresarios nacientes como a los empresarios que se encuentran establecidos hace tres años y medio. La variable dependiente binaria toma el valor de uno (1) si el joven se encuentra envuelto en alguna iniciativa de tipo empresarial, y cero (0) si no. Las variables independientes seleccionadas se limitaron a la información recopilada por la base de datos GEM, teniendo en cuenta los principales determinantes recopilados en los modelos de la evidencia empírica analizada.

\section{Resultados.}

Al tener una variable binaria, primero se procedió a evaluar el ajuste de los modelos Logit y Probit a la estructura de los datos. Para esto se corrieron ambos modelos y se seleccionó aquel que minimizará el AIC y el BIC según el criterio de información de Akaike y Schwarz, que en este caso es el modelo logístico (ver Anexo 1).

De los 465 individuos que componen la muestra se expulsaron 2 debido a limitaciones en las observaciones recopiladas para algunas variables. Los resultados generan evidencia que soporta la significancia global del conjunto de variables explicativas utilizadas (P-valor, Chi-Cuadrado $=$ 0.0000) dentro del modelo econométrico. Asimismo, la regresión fue sometida a las pruebas de bondad de ajuste (H-L), especificación y multicolinealidad (ver Anexos 2, 3 y 4), que demuestran la solidez del modelo. 


\begin{tabular}{|l|l|}
\hline Number of Obs & $=463$ \\
\hline LR Chi2 (17) & $=89.99$ \\
\hline Prob $>$ Chi2 & $=0.0000$ \\
\hline Pseudo R2 & $=\mathbf{0 . 1 7 1 2}$ \\
\hline Log likelihood & $=-\mathbf{2 1 7 . 8 1 1 3 3}$ \\
\hline
\end{tabular}

Tabla 4. Modelo Logit - Determinantes Emprendimiento Juvenil en Colombia, 2019.

\begin{tabular}{|c|c|c|c|c|c|c|}
\hline TEA & COEF. & STD. & $\mathbf{Z}$ & $\mathbf{P}>\mathbf{Z}$ & \multicolumn{2}{|c|}{ [95\% CONF. INTERVAL] } \\
\hline Suskil19 & .9822389 & 237107 & 4.14 & 0.000 & .5175178 & 144.696 \\
\hline Opport19 & .1001905 & .1941223 & 0.52 & 0.606 & -.2802822 & .4806632 \\
\hline Knowen19 & 1.164495 & .2835691 & 4.11 & 0.000 & .6087095 & 1.72028 \\
\hline Fearfail19 & -.9952638 & .2827903 & -3.52 & 0.000 & -1.549 .523 & -.4410049 \\
\hline Nbstat19 & .4466323 & .1786998 & 2.50 & 0.012 & .0963871 & .7968775 \\
\hline Nbgood19 & -.150088 & .1732304 & -0.87 & 0.386 & -.4896132 & 1894373 \\
\hline Gender & -.0205973 & .2458418 & -0.08 & 0.933 & -.5024384 & .4612438 \\
\hline \multicolumn{7}{|l|}{ Cohhinc } \\
\hline Between 1 and $2 S M M L V$ & .3389747 & .4184987 & 0.81 & 0.418 & -.4812676 & 1.159217 \\
\hline More than $2 S M M L V$ and less & .1296578 & .4721158 & 0.27 & 0.784 & -.7956722 & 1.054988 \\
\hline More than 3 SMMLV and less & -.4134425 & .5435716 & -0.76 & 0.447 & -1.478823 & .6519382 \\
\hline More than 4 SMMLV and less & -.5072515 & .7460425 & -0.68 & 0.497 & -1.969468 & .9549649 \\
\hline More than 5 SMMLV and less & .8483165 & 1.117944 & 0.76 & 0.448 & -1.342813 & 3.039446 \\
\hline \multicolumn{7}{|l|}{ Coreduc } \\
\hline Completed Secondary & .537959 & .5512106 & 0.98 & 0.329 & -.5423939 & 1.618312 \\
\hline Full Technician & .9827121 & .5901362 & 1.67 & 0.096 & -.1739335 & 2.139358 \\
\hline Technological Complete & 1.45669 & .6606241 & 2.21 & 0.027 & .1618905 & 2.751489 \\
\hline Full University & 1.071917 & .6830083 & 1.57 & 0.117 & -.2667543 & 2.410589 \\
\hline Postgraduate (Specializat..) & -.1316924 & 1.417932 & -0.09 & 0.926 & -2.910788 & 2.647404 \\
\hline Constant. & -3.21079 & .7517899 & -4.27 & 0.000 & -4.684 .271 & -1.737309 \\
\hline
\end{tabular}

Fuente: Elaboración propia.

Por su parte, la siguiente tabla muestra los efectos marginales promedio de la regresión logística. 
Tabla 5. Efectos Marginales Promedio de la Regresión Logística.

\begin{tabular}{|l|l|l|c|c|c|c|c|}
\hline $\begin{array}{l}\text { Marginal effects after logit } \\
\mathrm{y}=\text { (predict) } \\
=.10715608\end{array}$ \\
\hline \multicolumn{7}{|c|}{ EFECTOS MARGINALES } \\
\hline VARIABLE & \multicolumn{1}{|c|}{ DY/DX } & STD. ERR. & $\mathbf{Z}$ & $\mathbf{P}>\mathbf{Z}$ & [ 95\% C.I & X \\
\hline Suskil19 & .0939744 & .21901 & 0.43 & 0.668 & -.335287 & .523236 & .475162 \\
\hline Opport19 & .0095856 & .02843 & 0.34 & 0.736 & -.046144 & .065315 & .334773 \\
\hline Knowen19* & .1019281 & .03602 & 2.83 & 0.005 & .031322 & .172534 & .62419 \\
\hline Fairfail19* & -.0876651 & .03539 & -2.48 & 0.013 & -.157038 & -.018293 & .37149 \\
\hline Nbstat19 & .0427309 & .09967 & 0.43 & 0.668 & -.152619 & .238081 & .475162 \\
\hline Nbgood19 & -.0143595 & .03684 & -0.39 & 0.697 & -.086572 & .057853 & .481641 \\
\hline Gender & -.0019706 & .02439 & -0.08 & 0.936 & -.049776 & .045835 & 1.51404 \\
\hline
\end{tabular}

Fuente: Elaboración propia.

\subsection{Análisis de resultados.}

En concordancia con lo evidenciado en la literatura, para Colombia, la variable proxy ingreso, asociada al capital requerido para iniciar un negocio (Cohhinc), no es significativa dentro de los factores determinantes de la iniciativa empresarial por parte de los jóvenes, por lo que la hipótesis 1 se rechaza; sin embargo, tanto los rangos de ingreso más bajos (menos de 1 a 4 SMMLV) y el más alto (más de 6 SMMLV) tienen impactos positivos. Este hecho ayuda a generar la conclusión de que en Colombia el capital necesario para crear empresa no es visto como una barrera que disminuya dicha iniciativa por parte de los jóvenes. Dada la naturaleza categórica de la variable no se calcularon los efectos marginales de ésta.

La hipótesis 2 es rechazada debido a la no significancia estadística de la educación (Coreduc), lo que sigue la línea de Blanchflower (2004), quien encuentra que la educación tiene un impacto positivo en la actividad emprendedora sólo en los países desarrollados debido a la creación de empresas basadas en tecnologías, así como los aportes de Urrea et al. (2017) quien afirma que la formación se enfoca a formar empleados y no generadores de empleo e ingreso. Este hecho puede llegar a ser importante para la generación de políticas públicas, ya que apoya la idea de que las mejoras en los niveles de educación generan impactos positivos dentro de la probabilidad de generar autoempleos y, por ende, mejorar el bienestar socioeconómico de la población (Blanchflower \& Meyer, 1994; Rasheed, 2000; Turker \& Selcuk, 2008; Pihie, 2009; Montes \& Siga, 2009; Capaldo, 1997). En consecuencia, de acuerdo con Kantis (2018): "El sistema educativo debería en consecuencia ocupar un espacio central dentro de una estrategia de promoción del emprendimiento. El mercado no resuelve per se esta necesidad de atender desde edades tempranas la formación emprendedora de la población”. (p. 128)

La hipótesis 3 es rechazada. El modelo muestra que, aunque percibir oportunidades de negocio en su entorno (Opport19) tiene un efecto positivo en la probabilidad de ser un joven emprendedor, éste no es estadísticamente significativo. En promedio, la probabilidad de los individuos que son capaces de percibir oportunidades de negocio en el entorno aumenta en $9.39 \%$ con respecto a los que no.

La hipótesis 4 no es rechazada dado que los resultados para la variable miedo al fracaso (Fairfail19*), proxy de la percepción de riesgo, generan el signo esperado y tienen significancia dentro del modelo como determinante de la probabilidad de iniciativa empresarial por parte de los 
jóvenes. La probabilidad de ser emprendedor de un joven no adverso al riesgo con respecto uno adverso en promedio aumentan en $8.76 \%$.

La hipótesis 5 es aceptada. La variable autoconfianza (Suskil19), que da cuenta de la percepción de habilidades y conocimientos por parte de los individuos, tiene un impacto altamente significativo dentro de la probabilidad de que un joven sea emprendedor. De esta manera ante aumentos en la autoconfianza la probabilidad de ser un joven emprendedor en Colombia aumenta. Los resultados de la hipótesis 5 están en línea con los argumentos de Fairlie (2002); Wilson, Kickul, y Marlino (2007); Lafuente, Vaillant \& Gómez (2007); Olawale (2010); Capaldo (1997); Schiller \& Crewson (1997); Henderson y Robertson (1999); Wilson, Marlino y Kickul (2004). La probabilidad de ser emprendedor aumenta en $6.7 \%$ al percibir tener las habilidades y conocimientos para emprender con respecto a no hacerlo.

La hipótesis 6 es rechazada, en la medida que el coeficiente que acompaña a la variable género (Gender) es el esperado, pero no tiene significancia dentro del modelo. Lo que va en concordancia con las tendencias nacionales en relación al cierre de brechas entre hombres y mujeres (Gómez et al., 2017).

Por otro lado, la hipótesis 7 se acepta, ya que los resultados arrojan que la presencia de un marco de referencia donde alguien cercano haya iniciado un emprendimiento (Knowen19) impacta significativa y positivamente la probabilidad de ser emprendedor por parte de los jóvenes (Blanchflower \& Oswald, 1990; Schiller \& Crewson, 1997; Lafuente, Vaillant \& Gómez, 2007; Turker \& Selcuk, 2008; Pihie, 2009; Olmos \& Castillo, 2009). Esto puede deberse a que el contacto con otros emprendedores genera transferencias de conocimiento y habilidades basadas en la observación de las acciones de los otros emprendedores (Wood \& Bandura, 1989). Por otra parte, la probabilidad de ser un joven emprendedor aumenta en promedio en $5.67 \%$ para que aquellos que cuentan con alguien cercano que haya iniciado una empresa con respecto a los que no.

Por último, la hipótesis 8 es rechazada. La percepción del emprendimiento como una buena alternativa de carrera (Nbgood19) no fue estadísticamente significativa; sin embargo, la percepción de resultar un alto estatus de los empresarios (Nbstat19) sí fue significativa estadísticamente, esto va en contraposición de las proposiciones de Shapero y Sokol (1982), quienes demuestran que las sociedades con una alta valoración del emprendimiento tenderán a tener más empresarios, lo que no es reafirmado de forma completa por los resultados de este estudio. Sin embargo, esto se puede explicar con los hallazgos de los estudios GEM, en donde se exponen los altos niveles de actividad emprendedora y la alta valoración social del emprendimiento en Colombia, lo que sugiere que los efectos positivos de esta percepción pueden desvanecerse en el tiempo (Gómez et al., 2019). Aún así, es necesario destacar que, de acuerdo con un informe del Organismo Internacional para Juventud en Iberoamérica OJI (2018), "la juventud registra una alta percepción de aprovechar oportunidades (64\%) para iniciar un emprendimiento y manifiestan las ganas de iniciar un emprendimiento o negocios en los próximos tres años" (p. 81). Lo que significa que Colombia destaca como uno de los países donde los jóvenes tienen más intención de convertirse en emprendedores como consecuencia de los factores que se han descrito y analizado a lo largo de este trabajo.

\section{Conclusiones.}

En general, los resultados arrojados por el modelo son satisfactorios en el momento de explicar los posibles factores que determinan el emprendimiento juvenil en Colombia. Haciendo uso de las variables descriptivas utilizadas se concluye que la actitud de los jóvenes en Colombia en torno al emprendimiento, medida a través de las variables de autoconfianza, percepción de riesgo, percepción de oportunidades y valoración del emprendimiento como alternativa de carrera deseable, muestra una disposición positiva de éstos en relación con la iniciativa empresarial. 
Los resultados sugieren una relación significativa y positiva entre la tasa de actividad empresarial juvenil y la autoconfianza, los modelos de referencia y la percepción de oportunidades, hecho que evidencia que estos condicionantes informales expresados por Douglas North, son claves para influenciar la iniciativa empresarial por parte de los jóvenes en Colombia. La variable educación no fue determinante para explicar la decisión de crear una empresa, lo que se explica por los fallos del sector educativo, en donde la formación se enfoca a formar empleados y no generadores de empleo e ingreso.

De la significancia de la variable independiente del modelo de referencia, al igual que de los resultados de significancia arrojados por la variable explicativa educación, podemos destacar que la evidencia empírica sustenta la teoría económica institucional planteada por North (1993a), en donde se esboza que los códigos de conducta, las tradiciones, las reglas y las costumbres son determinantes en las decisiones tomadas por los individuos en el momento de decidir un emprendimiento.

En este caso, la variable conocer "a alguien cercano que haya decidido ser parte de una iniciativa empresarial" como proxy de los modelos de referencia puede ser tomado por los jóvenes de Colombia como un código de conducta o una base reguladora; como bien lo explica Bruton, Ahlstrom \& Li (2010) que, en ausencia de una normatividad, los jóvenes fijan directrices, influenciando las decisiones de hacer parte de actividades de creación de empresas. Por su parte, la variable educación puede ser tomada como un hábito o costumbre, que limita la capacidad de los jóvenes, afectando las acciones de éstos y la probabilidad de incorporación de los mismos en actividades empresariales.

Inesperadamente, variables como género y miedo al fracaso, no fueron significativas dentro del modelo, divergiendo de lo evidenciado en los estudios de Blanchflower y Meyer (1994), Blanchflower y Oswald (1998), Henderson (1999) y Wilson, Marlino y Kickul (2004), entre otros. En consideración de lo anterior, y siguiendo los preceptos teóricos de North (1993b) se puede concluir que las variables de miedo al fracaso, constituidas como condicionantes informales, pueden no ser vistas como una barrera que influencie la probabilidad de ingreso de los jóvenes colombianos en actividades emprendedoras.

Así mismo, por los resultados del modelo de probabilidad binaria Logit, podemos concluir que los condicionantes definidos por el enfoque de North (1993a), de tipo informal como la autoconfianza, modelo de referencia y percepción de oportunidades (Blanchflower \& Meyer, 1994; Blanchflower \& Oswald, 1998; Schiller \& Crewson, 1997, Fairlie, 2002; Delgado et al., 2007; Wilson, Kickul \& Marlino, 2007; Lafuente, Vaillant \& Gómez, 2007; Olawale, 2010; Capaldo, 1997) impactan mayormente la tasa de actividad empresarial de los jóvenes colombianos con respecto a los condicionantes de tipo formal; como el nivel de educación (Blanchflower \& Meyer, 1994; Rasheed, 2000; Turker \& Selcuk, 2008; Pihie, 2009; Montes \& Siga, 2009; Capaldo, 1997).

Se sugiere, que dada la evidencia arrojada por la variable nivel de educación (alto incompleto) y la argumentación de North (1993a), sobre la importancia de la creación de limitaciones formales que ayuden a disminuir los costes de búsqueda de información, la mejoría de este condicionante formal junto a la creación de incentivos que forjen a las instituciones a promover el desempeño económico vía educación, puede ser clave para mejorar la iniciativa empresarial de los jóvenes colombianos y por ende impactar positivamente en el bienestar socioeconómico experimentado por éstos.

Finalmente, los resultados demuestran que los planteamientos de la teoría económica institucional sobre emprendimiento se ajustan a la evidencia empírica contrastada a través del modelo econométrico utilizado. Si bien la identificación de los factores determinantes del emprendimiento juvenil en Colombia es significativa (toda vez que permite la formulación de políticas dirigidas a este segmento poblacional), existen otros elementos institucionales que hacen probables el surgimiento de numerosas iniciativas, a cargo de una variedad de partes interesadas, centradas en fomentar el emprendimiento entre los jóvenes colombianos. 


\section{Agradecimientos}

Este Artículo se realizó considerando la base de datos de Global Entrepreneurship Monitor, donde la Universidad del Norte es una de las instituciones fundadora para Colombia.

\section{Referencias}

Acs, Z.J., \& Amorós, J.E. (2008). Entrepreneurship and Competitiveness dynamics in Latin America. Small Business Economics, 31(3), 305-322. https://doi.org/10.1007/s11187-008-9133$\mathrm{y}$

Akaike, H. (1973). Maximum likelihood identification of Gaussian autoregressive moving average models. Biometrika, 60(2), 255-265. https://doi.org/10.2307/2334537

Aponte, M. (2002). Factores condicionantes de la creación de empresas en Puerto Rico. Un enfoque institucional. Tesis doctoral, Universidad Autónoma de Barcelona. https://www.tdx.cat/handle/10803/3946\#page=1

Arenius, P., \& Minniti, M. (2005). Perceptual variables and nascent entrepreneurship. Small Business Economics, 24(3), 233-247. https://doi.org/10.1007/s11187-005-1984-x

Baumol, W., Litan, R., \& Schramm, C. (2009). Good capitalism, bad capitalism, and the economics of growth and prosperity. New Haven, CT: Yale University Press. https://doi.org/10.1057/ces.2007.35

Blanchflower, D.G., \& Meyer, B.D. (1994). A longitudinal analysis of the young self-employed in Australia and the United States. Small Business Economics, 6(1), 1-19. https://doi.org/10.1007/BF01066108

Blanchflower, D., \& Oswald, A. (1990).What Makes a Young Entrepreneur? Documento 373, London School of Economics - Centro de Economía Laboral.

Blanchflower, D.G. (2004). Self-employment: More may not be better. Working Paper 10286. http://www.nber.org/papers/w10286

Blanchflower, D.G., \& Oswald, A.J. (1998). Entrepreneurship and the youth Labour market problem: A report for the OECD. Report to OECD, Paris.

Bosma, N., Acs, Z.J., Autio, E., Coduras, A., \& Levie, J. (2008). Global entrepreneurship monitor. Executive report. Global Entrepreneurship Research Association.

Bosma, N., Schøtt, T., Terjesen, S.A., \& Kew, P. (2016). Global entrepreneurship monitor 2015 to 2016: Special report on social entrepreneurship. Global Entrepreneurship Research Association. http://www.gemconsortium.org. https://dx.doi.org/10.2139/ssrn. 2786949.

Bruton, G.D., Ahlstrom, D., \& Li, H.L. (2010). Institutional theory and entrepreneurship: Where are we now and where do, we need to move in the future? Entrepreneurship theory and practice, 34(3), 421-440. https://doi.org/10.1111/j.1540-6520.2010.00390.x

Canales, G.R., Román S.Y., \& Ovando A.W. (2017). Emprendimiento de la población joven en México. Una perspectiva crítica. Entreciencias: Diálogos en la sociedad del conocimiento, 5(12). http://dx.doi.org/10.21933/J.EDSC.2017.12.211 
Capaldo, G. (1997). Entrepreneurship in southern Italy: empirical evidence of business creation by young founders. Journal of Small Business Management, 35(3), 86.

Chigunta, F.J. (2002). Youth entrepreneurship: Meeting the key policy challenges (pp. 1-34). Washington, D.C.: Education Development Center.

Coase, R.H. (1937). The nature of the firm. Economica, 4(16), 386-405.

Delgado, M.I., Gómez M.L., Romero, M.A.M., \& Vázquez, E. (2008). Determinantes sociales y cognitivos en el espíritu emprendedor: un estudio exploratorio entre estudiantes argentinos. Cuadernos de Gestión, 8(1), 11-24. https://www.redalyc.org/articulo.oa?id=274320547001

Fairlie, R.W. (2002). Drug dealing and legitimate self-employment. Journal of Labor Economics, 20(3), 538-537. https://doi.org/10.1086/339610

Fatoki, O. O. (2010). Graduate entrepreneurial intention in South Africa: Motivations and obstacles. International Journal of Business and Management, 5(9), 87-98. https:// /doi.org/10.5539/ijbm.v5n9p87

Formichella, M. (2004). El concepto de emprendimiento y su relación con la educación, el empleo y el desarrollo local. Buenos Aires, Argentina: Publicación INTA.

Foster, R. (1986). Innovation: The attacker's advantage. New York: Summit Books, New York.

Freytag, A., \& Thurik, R. (2007). Entrepreneurship and its determinants in a cross-country setting. Journal of Evolutionary Economics, 17(2), 117-131. https://doi.org/10.1007/s00191006-0044-2

García, P.O. (2016). Actitud emprendedora de los jóvenes españoles en un contexto de crisis. Revista de Ciencias Sociales, 22(1), 139-152. https://doi.org/10.31876/rcs.v22i1.24902

Global Entrepreneurship Monitor (GEM, 2016). GEM Colombia 2016. Universidad ICESI, Pontificia Universidad Javeriana de Cali, Universidad del Norte, Universidad EAN, Corporación Universitaria del Caribe, Universidad Cooperativa de Colombia de Bucaramanga.

Global Entrepreneurship Monitor (GEM, 2019). GEM Colombia 2019. Universidad Icesi, Pontificia Universidad Javeriana de Cali, Universidad del Norte, Universidad EAN, Corporación Universitaria del Caribe, Universidad Cooperativa de Colombia de Bucaramanga, Corporación Universitaria Americana.

Global Entrepreneurship Monitor (GEM, 2008). GEM Executive Report, 2008. Bosma, Z. J. Acs, E. Autio, A. Coduras, J. Levie, and Global Entrepreneurship Research Consortium (GERA). Babson College (Estados Unidos), Universidad del Desarrollo (Chile), London Business School (Reino Unido).

Gómez, L., López, S., Hernández, N., Galvis, M., Parra, L.D., Matiz, F.J.,... \& Martínez, P.C. (2019). GEM Colombia: estudio de la actividad empresarial en 2017. Universidad del Norte, Pontificia Universidad Javeriana de Cali, Universidad ICESI, Corporación Universitaria del Caribe-CECAR, Universidad Cooperativa de Colombia, Universidad EAN.

Guzmán, M.T.E., Landín, G.A., \& Saizarbitoria, I.H. (2007). Perfil emprendedor del alumnado universitario del campus de Gipuzkoa de la UPV/EHU. Revista de Dirección y Administración de Empresas, 14, 83-110. 
Hamilton, W.H. (1919). The institutional approach to economic theory. The American Economic Review, 9(1), 309-318.

Henderson, R., \& Robertson, M. (2000). Who wants to be an entrepreneur? Young adult attitudes to entrepreneurship as a career. Career Development International, 5(6), 279-287. https://doi.org/10.1108/13620430010373755

Kantis, H. (2016). La promoción del emprendimiento juvenil: Su importancia para América Latina. Pensamiento Iberoamericano, 1, 120-130.

Kirzner, I.M. (1973). Competition and entrepreneurship. Chicago: University of Chicago Press.

Levesque, M., \& Minniti, M. (2006). The effect of aging on entrepreneurial behavior. Journal of Business Venturing, 21(2), 177-194. https://doi.org/10.1016/j.jbusvent.2005.04.003

Makua, A., Amigo, M.C., \& del Valle, R.S.S. (2017). Relación entre las prácticas de ocio significativas de la juventud y el emprendimiento social.: El caso Think Big Jóvenes. OBETS: Revista de Ciencias Sociales, 12(1), 151-176. https://doi.org/10.14198/OBETS2017.12.1.16

Martínez, P., Gómez, L., Ramos, J., Arteaga, M., \& Caicedo, G. (2010). Fémale Entrepreneurship in Colombia Vs Ecuador, en espera para publicación.

Maxwell, J.R. (2002, January). Recommendations for connecting West African youth to entrepreneurship and small business through a multiple level strategy. In United States Association for Small Business and Entrepreneurship International Conference Reno, Nevada USA.

Ministerio de Educación Nacional. Ley 375 de Julio 4 de 1997. República de Colombia.

Montes, G.V., \& Siga, L. (2009). On the nature of micro-entrepreneurship: evidence from Argentina. Applied Economics, 41(21), 2667-2680. https://doi.org/10.1080/00036840701335553

Montes, V.J. (2016). Informe de emprendimiento juvenil en Colombia. https://www.incae.edu/sites/default/files/reporte_nacional_final__colombia_final_corregido.pdf

Nazri, M.A., Aroosha, H., \& Omar, N.A. (2016). Examination of Factors Affecting Youthsâ€ Entrepreneurial Intention: A Cross-Sectional Study. Information Management and Business Review, 8(5), 14-24. https://doi.org/10.22610/imbr.v8i5.1456

Nee, V., \& Young, F.W. (1991). Peasant entrepreneurs in China's" second economy": an institutional analysis. Economic Development and Cultural Change, 39(2), 293-310. https://doi.org/10.1086/451871

North, D.C. (1993a). Instituciones, cambio institucional y desempeño económico. Ciudad de México: Cambridge University Press y Fondo de Cultura Económico.

North, D.C. (1993b). Institutions and credible commitment. Journal of Institutional and Theoretical Economics (JITE)/Zeitschrift für die gesamte Staatswissenschaft, 149(1), 11-23.

North, D.C. (1994). Economic performance through time. The American Economic Review, 84(3), 359-368.

Olmos, R., \& Castillo, M. (2008). Factores explicativos sobre la actitud emprendedora de los estudiantes universitarios de la comunidad de Madrid España. In Anais XIX Congreso 
Latinoamericano y del Caribe sobre el Espíritu Empresarial, Florianópolis, Brasil, 21 a 23 de $\begin{array}{llll}\text { octubre } & \text { de } 2008 . & \text { Universidad }\end{array}$ http://bibliotecadigital.icesi.edu.co/biblioteca_digital/handle/item/1902

Organismo Internacional de Juventud para Iberoamérica (2018). Emprendimiento Juvenil en Iberoamérica: una clave para la empleabilidad. Madrid: Departamento de Comunicación.

Parada, J.J. (2003). Economía institucional original y nueva economía institucional: semejanzas y diferencias. Revista de Economía Institucional, 5(8), 92-116.

Pihie, Z.A.L., \& Akmaliah, Z. (2009). Entrepreneurship as a career choice: An analysis of entrepreneurial self-efficacy and intention of university students. European Journal of Social Sciences, 9(2), 338-349.

Rasheed, H. (2000). Developing entrepreneurial potential in youth of entrepreneurial education and venture creation. http://www. usasbe.org/knowledge/proceedings/2001/063. pdf.

Sahasranamam, S., \& Nandakumar, M. K. (2020). Individual capital and social entrepreneurship: Role of formal institutions. Journal of Business Research, 107, 104-117. https://doi.org/10.1016/j.jbusres.2018.09.005

Shah, I.A., Amjed, S., \& Jaboob, S. (2020). The moderating role of entrepreneurship education in shaping entrepreneurial intentions. Journal of Economic Structures, 9(1), 1-15. https://doi.org/10.1186/s40008-020-00195-4

Shapero, A., \& Sokol, L. (1982). The social dimensions of entrepreneurship. University of Illinois at Urbana-Champaign's Academy for Entrepreneurial Leadership Historical Research Reference in Entrepreneurship.

Schiller, B.R., \& Crewson, P.E. (1997). Entrepreneurial origins: A longitudinal inquiry. Economic inquiry, 35(3), 523-531.

Schoof, U. (2006). Stimulating Youth Entrepreneurship: Barriers and incentives to enterprise startups by young people. International Labour Organization.

Schumpeter, J. (1942). Capitalismo, socialismo y democracia. Barcelona: Ed. Folio.

De Soto, H. (2000). The mystery of capital: Why capitalism triumphs in the West and fails everywhere else. Basic Books.

Thomas, M.K. (2009). The impact of education histories on the decision to become self-employed: a study of young, aspiring, minority business owners. Small Business Economics, 33(4), 455-466.

Turker, D., \& Selcuk, S.S. (2009). Which factors affect entrepreneurial intention of university students? Journal of European industrial training, 33(2)142-159. https://doi.org/10.1108/03090590910939049

UNECE (2003).Youth in the UNECE Region: Realities. Challenges and Opportunities. Geneva: United Nations. Economic Commission for Europe.

Urbano D., Diaz, J., \& Hernández, R. (2007). Evolución y principios de la Teoría Económica Institucional. Una propuesta de aplicación para el análisis de los factores condicionantes de la Creación de Empresas. Investigaciones Europeas de Dirección y Economía de la Empresa, 13(3), 183-198. 
Urrea, F., Arango, L., Dávila, C., \& Parada, J. (2017). Innovación y Cultura de las Organizaciones en tres regiones de Colombia. Bogotá: Tercer Mundo, Corpocalidad y Colciencias.

Vaillant, Y., Gómez, E., \& Lafuente, E. (2011). El impacto de factores socio-culturales sobre la actividad emprendedora de los jóvenes en España. Cali: Universidad ICESI.

Veciana, J.M. (1988). Empresario y proceso de creación de empresas. Revista Económica de Cataluña, 8, 2-34.

Vergés, J.M.V. (1999). Creación de empresas como programa de investigación científica. Revista Europea de Dirección y Economía de la empresa, 8(3), 11-36.

Wilson, F., Kickul, J., \& Marlino, D. (2007). Gender, Entrepreneurial Self-Efficacy, and Entrepreneurial Career Intentions: Implications for Entrepreneurship. Education, Entrepreneurship Theory and Practice, 31(3), 387- 406.

Wilson, F., Marlino, D., \& Kickul, J. (2004). Our entrepreneurial future: Examining the diverse attitudes and motivations of teens across gender and ethnic identity. Journal of Developmental Entrepreneurship, 9(3), 177-197.

Wood, R., \& Bandura, A. (1989). Impact of conceptions of ability on self-regulatory mechanisms and complex decision making. Journal of Personality and Social Psychology, 56(3), 407-415. https://doi.org/10.1037/0022-3514.56.3.407

Yañez, R.C., \& Escareño, M.R. (2020). Política pública de fomento al emprendimiento juvenil para el desarrollo local en el municipio de Colima 2009-2013. SAPIENTIAE, 5(2), 328-342. 


\section{AneXos}

Anexo 1. Modelo Probit.

\begin{tabular}{|l|c|c|c|c|c|c|}
\hline \multicolumn{7}{|c|}{ Modelo Probit } \\
\hline TEA & COEF. & $\begin{array}{c}\text { STD. } \\
\text { ERR. }\end{array}$ & $\mathbf{Z}$ & $\mathbf{P}>\mathbf{Z}$ & \multicolumn{3}{|c|}{$\begin{array}{c}\text { [95\% CONF. } \\
\text { INTERVAL] }\end{array}$} \\
\hline Suskil19 & .5396641 & .1276296 & 4.23 & 0.000 & .2895147 & .7898136 \\
\hline Opport19 & .0550977 & .1134298 & 0.49 & 0.627 & -.1672206 & .2774161 \\
\hline Knowen19 & .7028334 & .1607141 & 4.37 & 0.000 & .3878395 & 1.017827 \\
\hline Fairfail19 & -.5665512 & .1589399 & -3.56 & 0.000 & -.8780677 & -.2550348 \\
\hline Nbstat19 & .2727901 & .1053988 & 2.59 & 0.010 & .0662123 & .4793679 \\
\hline Nbgood19 & -.0925721 & .09989 & -0.93 & 0.354 & -.2883529 & .1032087 \\
\hline Gender & -.0117974 & .1435466 & -0.08 & 0.934 & -.2931436 & .2695488 \\
\hline Cohhinc & & & & & & \\
\hline Between 1 and 2 SMMLV & .1738234 & .2334507 & 0.74 & 0.457 & -.2837315 & .6313783 \\
\hline More than 2 SMMLV and less & .033035 & .2656766 & 0.12 & 0.901 & -.4876816 & .5537516 \\
\hline More than 3 SMMLV and less & -.2473708 & .3041832 & -0.81 & 0.416 & -.8435588 & .3488173 \\
\hline More than 4 SMMLV and less & -.3194129 & .4210098 & -0.76 & 0.448 & -1.144577 & .5057511 \\
\hline More than 5 SMMLV and less & .4806355 & .6847351 & 0.70 & 0.483 & -.8614206 & 1.822692 \\
\hline Coreduc & & & & & & \\
\hline Completed Secondary & .2462937 & .2948532 & 0.84 & 0.404 & -.331608 & .8241954 \\
\hline Full Technician & .5146466 & .3190658 & 1.61 & 0.107 & -.1107109 & 1.140004 \\
\hline Technological Complete & .7925398 & .3624594 & 2.19 & 0.029 & .0821324 & 1.502947 \\
\hline Full University & .5398491 & .3828438 & 1.41 & 0.159 & -.2105109 & 1.290209 \\
\hline Postgraduate (Specializat) & -.2196999 & .8406275 & -0.26 & 0.794 & -1.867299 & 1.4279 \\
\hline Constant. & -1.793626 & .4056266 & -4.42 & 0.000 & -2.588639 & -.998612 \\
\hline & & & & & & \\
\hline & & & & & \\
\hline
\end{tabular}

\begin{tabular}{|l|c|c|c|c|c|c|}
\hline \multicolumn{7}{|c|}{ Modelo Logit } \\
\hline Model & Obs & LI (null) & LI (model) & df & AIC & BIC \\
\hline. & 463 & -262.804 & -2.178 .113 & 18 & 4.716 .227 & 5.461 .017 \\
\hline
\end{tabular}

\begin{tabular}{|l|c|c|c|c|c|c|}
\hline \multicolumn{7}{|c|}{ Modelo Probit } \\
\hline Model & Obs & Ll (null) & Ll (model) & df & AIC & BIC \\
\hline. & 463 & -262.804 & -2.179 .239 & 18 & 4.718 .479 & 546.327 \\
\hline
\end{tabular}

Fuente: Elaboración propia.

Anexo 2. Bondad de AJuste (H-L) Del Modelo Logístico.

\section{Logistic model for TEA, goodness-of-fit test}

(Table collapsed on quantiles of estimated probabilities)

\begin{tabular}{|l|c|}
\hline number of observations $=$ & 463 \\
\hline number of groups $=$ & 10 \\
\hline Hosmer-Lemeshow $\operatorname{chi} 2(8)=$ & 5.20 \\
\hline Prob $>$ chi $2=$ & 0.7361 \\
\hline
\end{tabular}

Fuente: Elaboración propia. 
ANEXO 3. ESPECIFICACIÓN DEL MODELO LOGÍSTICO.

\begin{tabular}{|l|c|c|c|c|c|c|}
\hline \multicolumn{7}{|c|}{ Specification link test for single-equation models } \\
\hline TEA & Coef. & Std. Err. & $\mathbf{z}$ & $\mathbf{P}>\mathbf{z}$ & \multicolumn{2}{|c|}{ [95\% Conf. Interval] } \\
\hline & & & & & & \\
\hline hat & 1.040633 & .24475 & 4.25 & 0.000 & .5609313 & 1.520334 \\
\hline hatsq & .0184331 & .0947205 & 0.19 & 0.846 & -.1672157 & .2040819 \\
\hline cons & .0042112 & .1571768 & 0.03 & 0.979 & -.3038496 & .3122721 \\
\hline
\end{tabular}

Fuente: Elaboración propia.

Anexo 4. VIF del Modelo Logístico.

\begin{tabular}{|c|c|c|}
\hline VARIABLES & VIF & 1/VIF \\
\hline Suskil19 & 1.71 & 0.585241 \\
\hline Opport19 & 1.44 & 0.694541 \\
\hline Knowen19 & 2.87 & 0.349024 \\
\hline Fairfail19 & 1.65 & 0.605145 \\
\hline Nbstat19 & 1.53 & 0.654275 \\
\hline Nbgood19 & 1.53 & 0.655283 \\
\hline Gender & 7.92 & 0.126209 \\
\hline \multicolumn{3}{|l|}{ Cohhinc } \\
\hline 2 & 4.55 & 0.219586 \\
\hline 3 & 2.44 & 0.410003 \\
\hline 4 & 2.03 & 0.493500 \\
\hline 5 & 1.45 & 0.689728 \\
\hline 6 & 1.14 & 0.878087 \\
\hline \multicolumn{3}{|l|}{ Coreduc } \\
\hline 4 & 5.76 & 0.173591 \\
\hline 6 & 2.80 & 0.356698 \\
\hline 8 & 1.94 & 0.514977 \\
\hline 10 & 1.79 & 0.557862 \\
\hline 11 & 1.22 & 0.819266 \\
\hline Mean VIF & 2.57 & \\
\hline
\end{tabular}

Fuente: Elaboración propia. 